\title{
Revalidação do método titulométrico para determinação do ácido ascórbico
}

\author{
Aline Inacio Alves ${ }^{1}$, Luciano José Quintão Teixeira², Sergio Henriques Saraiva ${ }^{3}$, Joel de Souza Carneiro ${ }^{4}$
}

\section{RESUMO}

Este estudo teve como objetivo revalidar o método de determinação de ácido ascórbico por titulometria, para ser aplicado no laboratório de Química de Alimentos, do Centro de Ciências Agrárias da Universidade Federal do Espírito Santo. O processo de revalidação foi realizado por meio da avaliação de parâmetros como linearidade, precisão (repetitividade), exatidão (teste de recuperação) e limites de detecção, considerando-se os critérios de aceitabilidade determinados. A significância adotada nos testes de hipóteses foi de 5\%. Foram realizadas análises de coeficiente de correlação linear simples (r), coeficiente de variação (CV) e a análise de regressão. Os dados foram analisados, utilizando-se o pacote estatístico Statistica 7.0. O método quantitativo por titulometria de ácido ascórbico foi revalidado e avaliado em amostras de ácido ascórbico e suco de limão. Verificou-se que o método está de acordo com os limites regulamentados pelos órgãos reguladores, sendo linear, repetitivo e com alta recuperação, apresentando um valor máximo de 98 e mínimo de 94,82\%, e um limite de detecção de $0,025 \mathrm{mg} \mathrm{mL}^{-1}$, no suco de limão.

Palavras-chave: titulação, validação intralaboratorial, análise de alimentos.

\section{ABSTRACT}

\section{Validation of the method for determination of ascorbic acid in lemon by titration}

The present study aimed to revalidate the method for determination of ascorbic acid by titration to be applied in the laboratory of Food Chemistry Centre of Agricultural Sciences, Federal University of Espírito Santo. The revalidation process was carried out by evaluating parameters such as linearity, precision (repeatability), accuracy (recovery test) and detection limits, considering the acceptability criteria determined. The level of significance in hypothesis testing was $5 \%$. Analyses included simple linear correlation coefficient (r), coefficient of variation (CV) and regression analysis. The data were analyzed using the software Statistica 7.0. The quantitative method by titration of ascorbic acid was revalidated and evaluated in samples of ascorbic acid and lemon juice. It was found that the same complies with the limits established by regulatory agencies. The methods presented linear, repetitive and high recovery, with a maximum value of $98 \%$ and a minimum of $94.82 \%$ and a detection limit of $0.025 \mathrm{mg} \mathrm{ml}^{-1}$ in the lemon juice.

Key words: titration, intralaboratory validation, food analysis.

\footnotetext{
Recebido para publicação em 27/03/2013 e aprovado em 25/10/2013.

${ }^{1}$ Engenheira de Alimentos. Universidade Federal de Viçosa, Campus Viçosa, Avenida Peter Henry Rolfs, s/n, 36570-000, Viçosa, Minas Gerais, Brasil. aline_inacio27@ @otmail.com. (autora para correspondência).

${ }^{2}$ Engenheiro de Alimentos, Doutor. Departamento de Engenharia de Alimentos, Universidade Federal do Espírito Santo, CP 16, 29500-000, Alegre, Espírito Santo, Brasil. luqteixeira@yahoo.com.br

${ }^{3}$ Engenheiro de Alimentos, Doutor. Departamento de Engenharia de Alimentos, Universidade Federal do Espírito Santo, CP 16, 29500-000, Alegre, Espírito Santo, Brasil. shsaraiva@yahoo.com.br

${ }^{4}$ Engenheiro de Alimentos, Doutor. Departamento de Engenharia de Alimentos, Universidade Federal do Espírito Santo, CP 16, 29500-000, Alegre, Espírito Santo, Brasil. joelcamilo@hotmail.com
} 


\section{INTRODUÇÃO}

A análise de alimentos apresenta necessidade crescente de métodos de ensaio que permitam atender aos constantes avanços tecnológicos na produção, processamento, embalagem e armazenamento de alimentos (Souza, 2007). Além dessa questão, os laboratórios de análises necessitam demonstrar competência técnica para garantir a qualidade dos ensaios que realizam (ISO, 2005), assegurando a confiabilidade e comparabilidade dos resultados que emitem, os quais, na maioria das vezes, subsidiam tomadas de decisão relativas a aspectos econômicos, de saúde pública (Brasil, 2006) e de defesa do consumidor.

Os laboratórios que analisam alimentos seguem, via de regra, normas de credenciamento estabelecidas por órgãos reguladores (Paschoal et al., 2008), os quais exigem que os métodos utilizados para um determinado objetivo sejam validados e, ou, revalidados. Os estudos para determinar os parâmetros de validação devem ser realizados com equipamentos e instrumentos dentro das especificações (INMETRO, 2007) e aplicam-se a métodos não normalizados; métodos criados ou desenvolvidos pelo próprio laboratório; métodos normalizados, usados fora do escopo para os quais foram concebidos, e ampliações e modificações de métodos normalizados.

A validação assegura a especificidade, exatidão e precisão de um ensaio analítico e estima a estabilidade do material analisado, durante a estocagem e manipulação. $\mathrm{O}$ método de validação de análises químicas é usado para suprir a necessidade de mostrar a qualidade dessas análises por meio de suas comparabilidade, rastreabilidade e confiabilidade (Lavra et al., 2008) sendo que, para tanto, deve apresentar seletividade, linearidade, precisão e exatidão (Silva \& Alves, 2006).

A validação de métodos tem representado uma etapa importante para os laboratórios de análises de alimentos e, consequentemente, um obstáculo para a credibilidade dos resultados emitidos (King, 2003). Todo método, antes da sua utilização, deve ser validado no laboratório em que será aplicado, a fim de assegurar as condições mínimas de sua execução com a confiabilidade requerida (Pereira et al., 2007).

A validação deve garantir, por meio de estudos experimentais, que o método atenda às exigências das aplicações analíticas (Ribani et al., 2004). Ter um resultado obtido por um método validado significa que o procedimento, que inclui desde as condições de operação do equipamento até toda a sequência analítica, seja aceito como correto (Silva \& Alves, 2006).

Diante do exposto, este trabalho objetivou revalidar o método de determinação de ácido ascórbico por titulometria, para ser aplicado no laboratório de Química de Alimentos, do Centro de Ciências Agrárias, da Universidade Federal do Espírito Santo. O processo de revalidação foi realizado por meio da avaliação de parâmetros como linearidade, precisão (repetitividade), exatidão (teste de recuperação) e limites de detecção.

\section{MATERIAL E MÉTODOS}

\section{Determinação de ácido ascórbico}

A determinação do teor de ácido ascórbico foi realizada pelo Método oficial 967.21, da Association of Official Analytical Chemists (AOAC, 1998), o qual consiste em reduzir uma substância colorida, 2,6- diclorofenolindofenol, em titulação com uma solução contendo ácido ascórbico, sendo as soluções preparadas pouco antes do uso e, o ácido ascórbico, preparado imediatamente antes do uso.

\section{Revalidação}

Os parâmetros estudados para revalidação foram: linearidade, exatidão, precisão e limite de detecção, considerando-se os critérios de aceitabilidade determinados. Adotou-se a significância de 5\% nos testes de hipóteses. Os dados foram analisados com o pacote estatístico Statistica 7.0.

\section{Linearidade}

A linearidade do método foi avaliada, utilizando-se análise de regressão linear e a análise de correlação entre a concentração e o volume de titulação. $\mathrm{O}$ método foi baseado no apresentado pelo INMETRO (2007).

O coeficiente de correlação linear simples (r) e a análise de regressão que é representada por uma equação da reta que relaciona as duas variáveis:

$y=a+b x$

Eq. 1

em que: $\mathrm{y}=$ resposta (medida do volume do titulante); $\mathrm{x}=$ concentração de ácido ascórbico; a = interseção com o eixo $y$, quando $x=0 ; b=$ inclinação da curva analítica.

Foram testadas sete diferentes concentrações, de forma a se elevar a confiabilidade dos resultados obtidos para esse parâmetro. Para a análise de linearidade do ácido ascórbico, foram utilizadas as concentrações de: 0,$5 ; 1,0$; 2,0; 3,0; 4,0; 5,0 e 6,0 $\mathrm{mg} \mathrm{ml}^{-1}$. Para o suco de limão, foram utilizados: 0,$1 ; 0,5 ; 1,0 ; 1,5 ; 2,0 ; 2,5$ e 3,0 mL. O volume e a concentração foram determinados por testes preliminares.

Para determinar a quantidade de ácido ascórbico, nas amostras de suco de limão, realizou-se uma análise de regressão, relacionando-se o volume gasto de titulante com a concentração de ácido ascórbico presente nas amostras analisadas. Foram realizadas três repetições para cada nível de concentração e volume.

\section{Exatidão}

A determinação da exatidão foi realizada por ensaio de recuperação, a partir de análises de amostras adicionadas 
de concentrações conhecidas de ácido ascórbico, em três repetições.

A amostra fortificada foi preparada, utilizando-se solução de suco de limão, com volume de ácido ascórbico pré-estabelecido na análise de linearidade. As amostras de suco de limão foram fortificadas com as seguintes concentrações do material analisado (ácido ascórbico): 0,2; 0,$5 ; 0,7 ; 1,0$ e $2,0 \mathrm{mg} \mathrm{mL}^{-1}$.

A recuperação $(\mathrm{R})$ foi calculada segundo a equação 2 :

$\mathrm{R}(\%)=((\mathrm{C} 1-\mathrm{C} 2) / \mathrm{C} 3) \times 100$

Eq. 2

em que: $\mathrm{C} 1$ = concentração do ácido ascórbico na amostra fortificada, C2 = concentração do ácido ascórbico na amostra não fortificada, C3 = concentração do ácido ascórbico adicionado à amostra fortificada. $\mathrm{O}$ método foi baseado no apresentado pelo INMETRO (2007).

\section{Precisão}

A precisão foi avaliada quanto à repetitividade que foi determinada pela análise de sete repetições. As concentrações utilizadas foram: 0,$5 ; 1,0 ; 1,5$ e 2,0 mg.mL $\mathrm{mL}^{-1}$ de ácido ascórbico e 0,$5 ; 1,0 ; 1,5$ e 2,0 mL de suco de limão.

$\mathrm{O}$ coeficiente de variação $(\mathrm{CV})$, também conhecido como desvio padrão relativo (DPR), foi calculado pela equação:

$\mathrm{C} . \mathrm{V}=\mathrm{DPR}=\mathrm{DP} / \mathrm{CMD} \mathrm{X} 100$

em que: $\mathrm{DP}=$ desvio padrão; $\mathrm{CMD}=$ concentração média determinada.

\section{Limite de detecção}

O limite de detecção foi estabelecido por meio da análise de soluções de concentrações conhecidas e decrescentes do material analisado, até o menor nível detectável. O procedimento foi realizado com base em Eurachem Working Group (1998).

Foram analisados os seguintes volumes de suco de limão: 2,5; 2,0; 1,5; 1,0; 0,5; 0,1;0,09; 0,05; 0,03 e 0,01 mL, com os quais foram calculadas as suas respectivas concentrações de ácido ascórbico, obtidas na titulação. Os resultados foram analisados para determinação do limiar de perda de confiabilidade do método, ou seja, do ponto em que o método passa a apresentar resultados não detectados para amostras. Nesse caso, o menor nível de concentração estudado, no qual o material analisado foi detectado para todas as repetições, foi considerado como limite de detecção do método.

\section{RESULTADOS E DISCUSSÃO}

\section{Linearidade}

Na Figura 1a, por meio da análise de regressão, é possível observar que, para o ácido ascórbico, o método apre- sentou linearidade no intervalo das concentrações testadas, apresentando coeficiente de determinação $\left(\mathrm{R}^{2}\right)$ de 0,999. Este parâmetro indica baixa dispersão do conjunto de pontos experimentais e reduzida incerteza dos coeficientes de regressão estimados. Para Ribani et al. (2004), quanto mais próximo de um é o $\mathrm{R}^{2}$, maior é a qualidade da curva obtida e maior a confiabilidade dos métodos analíticos.

O mesmo comportamento foi observado para o ácido ascórbico, quantificado nas diferentes diluições do suco de limão (Figura 1b), cujo modelo apresentou linearidade.

O coeficiente de correlação foi calculado, utilizandose o coeficiente de correlação linear simples a 5\% de probabilidade. Nos dois casos, o valor do $r$ foi superior a 0,99 , demonstrando correlação entre o volume titulado e a concentração.

Estes resultados estão dentro dos limites impostos pelas principais agências reguladoras nacionais. A Agência Nacional de Vigilância Sanitária - Anvisa, por meio da sua resolução 899 de 2003 (ANVISA, 2003), recomenda um coeficiente de correlação igual a 0,99 para se considerar um método como adequado, enquanto, o Instituto Na-
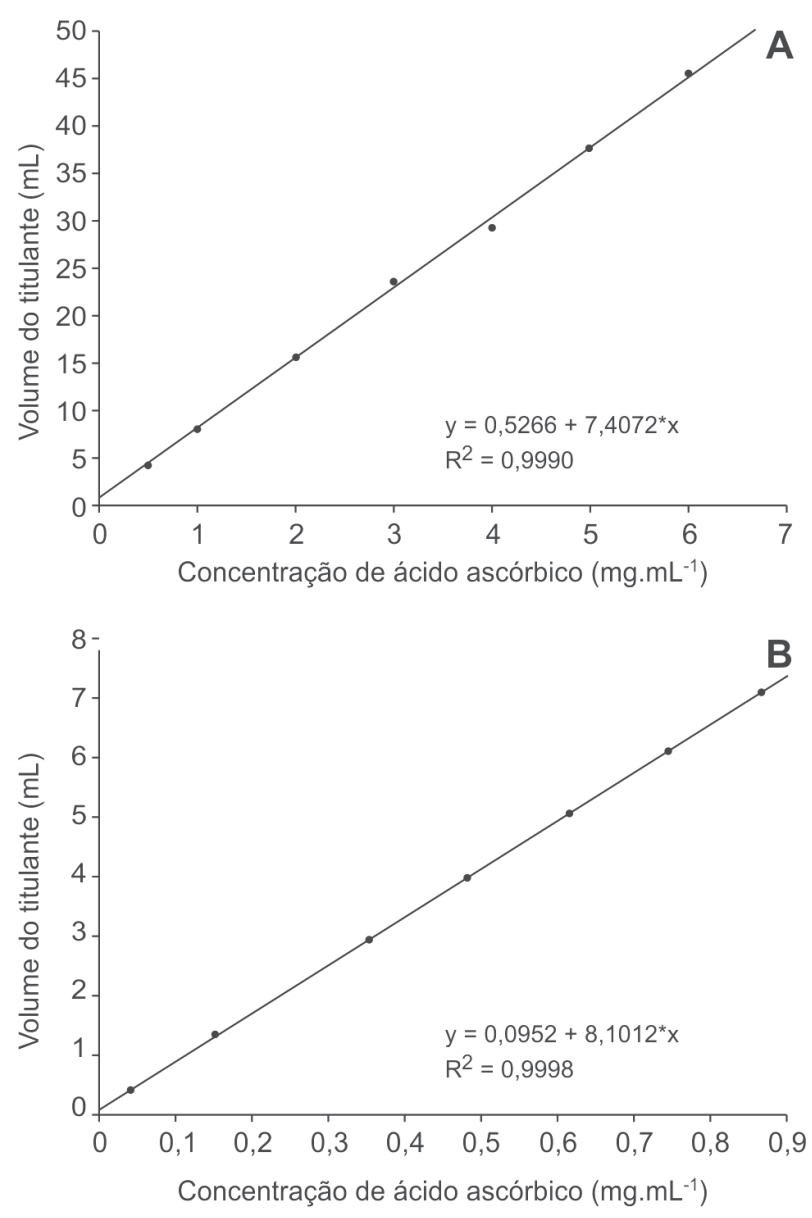

Figura 1. Modelo de regressão linear ajustado (A) para o ácido ascórbico; (B) para o ácido ascórbico presente nas amostras de suco de limão.

Rev. Ceres, Viçosa, v. 61, n.2, p. 155-161, mar/abr, 2014 
cional de Metrologia, Normalização e Qualidade Industrial - INMETRO, admite a linearidade de um método, desde que este apresente valores de correlação acima de 0,90 (INMETRO, 2007).

Cardoso et al. (2010), trabalhando com a validação de métodos para determinação de resíduos de agrotóxicos em tomate, encontraram coeficientes de determinação superiores a 0,95 e de correlação superiores a 0,98, aceitando-os como satisfatórios na indicação de linearidade para os métodos testados.

Vale ressaltar que, em qualquer técnica instrumental, a relação linear simples, descrita pela equação da reta, só é válida em um determinado intervalo de concentrações da espécie medida, não sendo possível a extrapolação dos resultados para valores que estejam fora desse intervalo. Barros Neto et al. (2002) denominam este intervalo de massas ou de concentrações, no qual se pode construir uma curva analítica linear, de faixa linear dinâmica, e afirmam que ele deve ser o mais extenso possível, ou seja, que a distância entre os pontos (distância nos valores de concentração) seja razoável.

\section{Precisão}

Para a precisão do método, foi calculado o desvio padrão e o desvio padrão relativo de cada conjunto de determinações, sendo os resultados obtidos expostos nas Tabelas 1 e 2.

Os valores para o coeficiente de variação, tanto para o método utilizando ácido ascórbico quanto para o método utilizando o limão, foram baixos, sendo o maior $(4,98)$ observado para o volume de 2,0 mL de amostra de suco de limão. Considerando-se o coeficiente de variação inferior, de 5\% de variação, apresentado pela RE 899 (ANVISA, 2003), esses resultados evidenciam a precisão dos métodos com boa repetitividade, tanto utilizando-se o suco de limão quanto o ácido ascórbico na titulação. Siqueira-Moura et al. (2008), em trabalho com validação de método analíti- co para determinação de ácido úsnico, em lipossomas, encontraram repetitividade com coeficiente de variação de $1,96 \%$.

Outros autores, como Roudaut (1998) e Schenck \& Lagman (1999), encontraram, para algumas amostras, variação superior a 5\% (algumas atingindo valores de 23,7\%). Apesar disso, consideraram os seus métodos precisos, uma vez que obtiveram médias de recuperação aparente de amostra, superior a 90\%, níveis dentro do recomendado pela Codex Alimentarius (Codex Alimentarius Comission, 2003). Souza et al. (2007), porém, afirmam que variações nessa escala $(\mathrm{CV}>5 \%)$ comprometem significativamente o método, uma vez que existe a possibilidade de incorrer em erros, dada a sua baixa precisão.

Independentemente do critério de aceitabilidade utilizado, os resultados obtidos neste trabalho são categóricos quanto à precisão do método. Desde que se garantam as condições experimentais, os resultados apresentarão baixa variação, o que indica a adequação do método para uso nas atividades de determinação de ácido ascórbico.

\section{Exatidão}

Os resultados obtidos na análise de recuperação e as respectivas concentrações de ácido ascórbico adicionadas em uma base de suco de limão com concentração conhecida são mostrados na Tabela 3.

A percentagem de recuperação aceitável depende da concentração e de requisitos do nível de qualidade requeridos. No método de titulometria para determinação de ácido ascórbico, obteve-se um nível de recuperação variando de 94,82 a 98\%, em função da concentração de ácido ascórbico adicionado às amostras de suco de limão. Considerando-se que em análises de alimentos, a Food and Drug Administration (FDA) recomenda faixas de 80 a $110 \%$ para concentrações do material analisado acima de $0,1 \mathrm{mg} \mathrm{kg}^{-1}$ e de 60 a $100 \%$, para concentrações

Tabela 1. Desvio padrão e coeficiente de variação sob condições de repetitividade para o ácido ascórbico

\begin{tabular}{lccc}
\hline Concentração $(\mathbf{m g} / \mathbf{m L})$ & Volume de titulante $(\mathbf{m L})$ & Desvio Padrão & Coeficiente de variação $(\%)$ \\
\hline 0,5 & 1,39 & 0,13 & 1,58 \\
1,0 & 2,97 & 0,14 & 0,90 \\
1,5 & 3,91 & 0,36 & 1,55 \\
2,0 & 5,07 & 0,15 & 0,51 \\
\hline
\end{tabular}

Tabela 2. Desvio padrão e coeficiente de variação sob condições de repetitividade para o suco de limão

\begin{tabular}{lccc}
\hline Suco de Limão $(\mathbf{m L})$ & Volume de titulante $(\mathbf{m L})$ & Desvio Padrão & Coeficiente de variação $(\%)$ \\
\hline 0,5 & 7,93 & 0,049 & 0,96 \\
1,0 & 15,44 & 0,090 & 2,30 \\
1,5 & 23,11 & 0,049 & 1,64 \\
2,0 & 29,27 & 0,069 & 4,98 \\
\hline
\end{tabular}

Rev. Ceres, Viçosa, v. 61, n.2, p. 155-161, mar/abr, 2014 
abaixo de $0,1 \mathrm{mg} \mathrm{kg}^{-1}$ (Bruce et al., 1998), os resultados obtidos neste trabalho confirmam a exatidão do método utilizado.

Segundo Ribani et al. (2004), para recuperação, os intervalos aceitáveis para análise de resíduos geralmente estão entre 70 e $120 \%$, com precisão de até $\pm 20 \%$. Dependendo da complexidade analítica e da amostra, este valor pode ser de 50 a $120 \%$, com precisão de até $\pm 15 \%$.

A European Commission (2002) determina faixas de recuperação médias, em análises de resíduos em alimentos, sendo de 50 a $120 \%$, para níveis de recuperação $\geq 1$ $\mu \mathrm{g} / \mathrm{kg}$, de 70 a $110 \%$, para níveis de concentração > $1 \mu \mathrm{g} /$ $\mathrm{kg}$ a $10 \mathrm{ìg} / \mathrm{kg}$ e, de 80 a 11 0\%, para níveis de concentração $\geq 10 \mu \mathrm{g} / \mathrm{kg}$.

Souza (2007), utilizando o método de cromatografia líquida de alta eficiência, para determinar semicarbazida em amostras de purê de maçã, refeição de carne e pudim de arroz, encontraram médias de recuperação variando de 93,8 a $107,2 \%$; de 96,6 a $104,1 \%$ e de 87,8 a $112,9 \%$, respectivamente.

Em um trabalho utilizando a metodologia LC-MS/MS, para determinação simultânea das aflatoxinas AFB1, AFB2, AFG1 e AFG2 em castanha-do-brasil, Teixeira (2008) encontrou recuperações variando entre 92 e $100 \%$, para amostras com um nível de contaminação entre 1,2 e 11,5 ìg/kg. Esse autor considera como melhor que o método de cromatografia de alta eficiência a metodologia avaliada, a qual obteve valores de recuperação de 93, 97, 95, e 95\% para AFG1, AFB1, AFG2 e AFB2, respectivamente em amostras de castanha-do-brasil.

Aldrigue (1998), avaliando diversos modos de se determinar ácido ascórbico em polpa de acerola, concluiu que o método de Tilmans foi o que apresentou os melhores resultados, quando esses foram avaliados pela percentagem de recuperação. Para esse autor, o método pode ser considerado o mais eficiente para a determinação de ácido ascórbico em amostras de frutas. Oliveira et al. (2010), entretanto, ressalvam que, apesar da eficiência, o método de Tilmans apresenta dificuldades quando são analisadas amostras de intensa coloração, como no caso de algumas frutas, geleias, sucos e outros. Isso se dá, conforme Aldrigue (1998), pela coloração natural da amostra que dificulta a visualização do ponto final.

Tabela 3. Médias de recuperação das concentrações de ácido ascórbico analisadas

\begin{tabular}{lc}
\hline Concentração (mg/mL) & Recuperação $(\%)$ \\
\hline 0,2 & 95,00 \\
0,5 & 98,00 \\
0,7 & 97,85 \\
1,0 & 96,76 \\
2,0 & 94,82 \\
\hline
\end{tabular}

\section{Limite de detecção}

$\mathrm{Na}$ Tabela 4, são apresentados os resultados detectados e não detectados, em função do nível de concentração de material analisado. Em seguida, os dados da Tabela são analisados para determinação do limiar de perda de confiabilidade do método, ou seja, do ponto em que o método passa a apresentar resultados não detectados para amostras adicionadas.

O limite de detecção foi determinado visualmente e, dessa maneira, foi considerado como sendo o menor volume, que contém a menor concentração da solução que o método distingue, com segurança. Souza (2007) afirma que não existe um método ótimo para determinação de limites de detecção, sendo aceitáveis os mais diversos utilizados com essa finalidade.

Pode ser observado, pelos dados obtidos, que o limite de detecção de ácido ascórbico encontra-se entre os valores de 0,037 a $0,025 \mathrm{mg} / \mathrm{mL}$, sendo considerado como limite de detecção para ácido ascórbico, no suco de limão, um volume de $0,09 \mathrm{~mL}$, o que significa que, para volumes inferiores a este, o método não consegue identificar a presença do ácido ascórbico na solução. Paschoal et al. (2008) afirmam que, em termos gerais, o limite de detecção indica o limite em que a menor quantidade que pode ser detectada do material analisado, em uma amostra, embora essa quantidade não possa ser quantificada.

Teixeira (2008) utilizou a análise de cromatogramas, para dar origem ao limite de detecção, que foi determinado pela visualização da menor concentração visível (detectável) para cada aflatoxina, sendo de 0,263; 0,345; 0,241 e $0,254 \mathrm{mg} / \mathrm{kg}-1$ de amostra para AFB1,AFB2, AFG1 e AFG2, respectivamente.

Os resultados apresentados encontram-se próximos aos observados por diversos autores que realizaram avaliações com vitaminas. Giacomini (2006), na detecção de vitamina A em polivitamínicos, utilizando cromatografia, encontrou o limite de detecção a partir da concentração

Tabela 4. Resultados referentes à análise do limite de detecção do material analisado detectado e não detectado

\begin{tabular}{lcc}
\hline $\begin{array}{c}\text { Suco Limão } \\
(\mathbf{m L})\end{array}$ & $\begin{array}{c}\text { Volume de } \\
\text { titulante } \\
(\mathbf{m L})\end{array}$ & $\begin{array}{c}\text { Concentração de } \\
\text { ácido ascórbico } \\
(\mathbf{m g} / \mathbf{m L})\end{array}$ \\
\hline 2,5 & 6,1 & 0,744 \\
2,0 & 5,03 & 0,612 \\
1,5 & 3,9 & 0,47 \\
1,0 & 3,0 & 0,36 \\
0,5 & 1,3 & 0,149 \\
0,1 & 0,4 & 0,037 \\
0,09 & 0,3 & 0,025 \\
0,05 & 0,3 & 0,025 \\
0,03 & 0,3 & 0,025 \\
0,01 & 0,3 & 0,025 \\
\hline
\end{tabular}

Rev. Ceres, Viçosa, v. 61, n.2, p. 155-161, mar/abr, 2014 
de $0,47 \mathrm{mg} / \mathrm{mL}$. De acordo com esse autor, o pico do cromatograma da solução de vitamina A destacou-se, situando-se a uma altura três vezes superior à da média das oscilações de ruído da linha de base cromatográfica. Quian \& Sheng (1998), também estudando vitamina A, encontraram limite de detecção de $0,10 \mathrm{mg} / \mathrm{mL}$ em amostra de ração. Stefanon (2001), avaliando simultaneamente as vitaminas A, D3 e E, em multivitamínicos, encontrou limites de detecção de $0,7 \mathrm{mg} / \mathrm{mL}, 0,11 \mathrm{mg} / \mathrm{mL}$ e $0,125 \mathrm{mg} / \mathrm{mL}$, respectivamente.

\section{CONCLUSÕES}

A revalidação da análise mostrou-se bastante eficiente e útil, por meio da avaliação do desempenho quanto à linearidade, precisão, exatidão e limite de detecção.

A linearidade, para as concentrações de ácido ascórbico em suco de limão, apresentou coeficientes de correlação dentro dos recomendados pelos principais órgãos oficiais de regulamentação.

Os resultados evidenciaram a precisão do método, com boa repetitividade, considerando-se o limite de variação regulado pela Anvisa. A exatidão apresentou resultados satisfatórios, dentro dos padrões da FDA.

A revalidação da metodologia de determinação de ácido ascórbico por titulometria confirmou ser, esta, uma técnica precisa e confiável, com vantagens significativas na determinação desse composto, assegurando a confiabilidade e comparabilidade dos resultados emitidos e permitindo que o laboratório garanta sua competência técnica e a qualidade dos ensaios que realiza.

\section{REFERÊNCIAS}

Aldrigue ML (1998) Desenvolvimento e validação de metodologia analítica, utilizando a CLAE, para determinação de ácido ascórbico em frutas e seus principais produtos. Tese de Doutorado. Universidade Estadual de Campinas, Campinas. 182p.

ANVISA - Agência Nacional de Vigilância Sanitária (2003) Resolução RE 899, de 29 de maio de 2003. Guia para a validação de métodos analíticos e bioanalíticos. Disponível em: <http:// elegis.anvisa.gov.br/leisref/public/showAct.php?id=15132\&word =legislacao>. Acessado em: 17 de março de 2013.

AOAC - Association of Official Analytical Chemists (1998) AOAC Peer-verified methods program. Manual on polices and procedures. Gaithersburg, AOAC. 35p.

Barros Neto B, Pimentel MF \& Araujo MCU (2002) Recomendações para calibração em química analítica: parte I. Fundamentos e calibração com um componente (calibração univariada). Química Nova, 25:856-865.

BRASIL (2006) Ministério da Agricultura, Pecuária e Abastecimento. Portaria número 50 de 20 de fevereiro de 2006. Aprova os Programas de Controle de Resíduos em Carne, Leite, Mel, Ovos e Pescado do exercício de 2006. Disponível em: <http:// www.agricultura.gov.br/arq_editor/file/CRC/PORTARIA\%2050\% 202006.pdf >. Acessado em: 15 de março de 2013.
Bruce B, Minkkinen P \& Riekkola ML (1998) Practical method validation: validation sufficient for an analysis method. Acta, 128:93-106.

Cardoso MHWM, Gouvêa AV, Nóbrega AW \& Abrantes SMP (2010) Validação de método para determinação de resíduos de agrotóxicos em tomate: uma experiência laboratorial. Ciência e Tecnologia de Alimentos, 30:63-72.

Codex Alimentarius Comission (2003) Guidelines on Good Laboratory Practice in Residue Analysis: CAC/GL 40-1993. Disponível em: <http://www.google.com.br/url?sa=t\&rct= $\mathrm{j} \& \mathrm{q}=$ guidelines $\% 20 \mathrm{on} \% 20 \operatorname{good} \% 201$ aboratory $\% 20$ practice $\%$ 20in\%20residue\%20analysis $>$. Acessado em: 17 de março de 2013.

European Commission (2002) Implementing Council Directive 96/23/EC concerning performance of analytical methods and the interpretation of results. Disponível em: <http:// www.fas.usda.gov/ffpd/WTO_SPS_TBT_Notifications/ Fishery_Products/EU219txt.pdf $>$. Acessado em: 17 de março de 2013

Eurachem Working Group (1998) The fitness for purpose of analytical methods, a laboratory guide to method validation and related topics. Teddington, LGC. 61p.

Giacomini LZ (2006) Quantificação de vitamina a em concentrados polivitaminicos por cromatografia líquida de alta eficiência. Dissertação de Mestrado. Universidade Federal de Santa Maria, Santa Maria. 64p.

INMETRO - Instituto Nacional de Metrologia, Normalização e Qualidade Industrial (2007) Orientações sobre Validação de Métodos de Ensaios Químicos, DOQ-CGCRE-008. Disponível em: <http://www.inmetro.gov.br/Sidoq/Arquivos/CGCRE/ DOQ/DOQ-CGCRE-8_03.pdf>. Acessado em: 17 de março de 2013.

ISO - International Standard Organization (2005) ISO/IEC 17025: General requirements for the competence of testing and calibration laboratories. Disponível em: <http://webstore.iec.ch/ preview/info_isoiec17025\%7Bed2.0\%7Den.pdf $>$. Acessado em: 17 de março de 2013.

King B (2003) In-house method validation. A guide for chemical laboratories. LGC Limited. Disponível em: <http:// www.nmschembio.org.uk/PublicationArticleaspx $\quad$ m= 115\&amid=1532>. Acessado em: 15 de março de 2013.

Lavra ZMM, Neto PJR, Silva RMF \& Medeiros FPM (2008) Desenvolvimento e validação de método analítico para determinação simultânea de Lamivudina, zidovudina e nevirapina em comprimidos dose-fixa combinada por cromatografia líquida de alta eficiência. Química Nova, 31:969-974.

Oliveira RG, Godoy HT \& Prado MA (2010) Otimização de metodologia colorimétrica para a determinação de ácido ascórbico em geleias de frutas. Ciência e Tecnologia de Alimentos, 30:244-249.

Paschoal JAR, Rath S, Airoldi FPS \& Reyes FGR (2008) Validação de métodos cromatográficos para a determinação de resíduos de medicamentos veterinários em alimentos. Química Nova, 31:1190-1198.

Pereira A, Scheshowitsch K, Cruz A, Silva MAS \& Stulzer HK (2007) Validação de metodologia analítica para quantificação de piroxicam em cápsulas de gelatina por espectrofotometria ultravioleta (UV). Visão Acadêmica, 8:29-37.

Quian H \& Sheng M (1998) Silmutaneous determination of fatsoluble vitamins A, D and E and pro-vitamin D2 in animal feeds by one-step extraction and high-performance liquid chromatography analysis. Journal of Chromatography Analysis, $825: 127-133$. 
Ribani M, Bottoli CBG, Collins CH, Jardim ICSF \& Melo LFC (2004) Validação em métodos cromatográficos e eletroforéticos. Química Nova, 27:771-780.

Roudaut B (1998) Multiresidue method for the determination of avermectin and moxidectin residues in the liver using HPLC with fluorescence detection. Analyst, 123:2541-2544.

Schenck FJ \& Lagman LH (1999) Multiresidue determination of abamectin, doramectin, ivermectin, and moxidectin in milk using liquid chromatography and fluorescence detection. Journal of AOAC International, 82:1340-1344.

Silva AP \& Alves MCC (2006) Como iniciar a validação de métodos analíticos. In: Congresso e Feira da Qualidade em Metrologia Rede Metrológica do Estado de São Paulo, São Paulo. Anais, Remesp- Rede Metrológica do Estado de São Paulo. 1:8-15.

Siqueira-Moura MP, Lira MCB \& Santos-Magalhães NS (2008) Validação de método analítico espectrofotométrico UV para determinação de ácido úsnico em lipossomas. Revista Brasileira de Ciências Farmacêuticas, 44:621-628.

Souza SVC (2007) Procedimento para validação Intralaboratorial de métodos de ensaio: Delineamento e aplicabilidade em análises de alimentos. Tese de Doutorado. Universidade Federal de Minas Gerais, Belo Horizonte. 296p.
Souza SVC, Lima JA, Teodoro JC \& Junqueira RG (2007) Validação intralaboratorial de método quantitativo para determinação múltipla de resíduos de avermectinas em leite bovino por cromatografia líquida de alta eficiência com detecção de fluorescência. Ciência e Tecnologia de Alimentos, 27:823-836.

Stefanon EBC (2001) Determinação simultânea das vitaminas A, D3, E e da vitamina K3 em multivitamínicos utilizando cromatografia liquida de alta eficiência. Dissertação de Mestrado. Universidade Federal de Santa Maria, Santa Maria. 213p.

Teixeira AS (2008) Adequação e apresentação de parâmetros de validação intralaboratorial de um ensaio para a quantificação de Aflatoxinas em Castanha-dobrasil (Bertholletia excelsa Bonpl.) através de Cromatografia Líquida de Alta Eficiência. Dissertação de Mestrado. Universidade Federal do Rio de Janeiro, Rio de Janeiro. $143 \mathrm{p}$. 